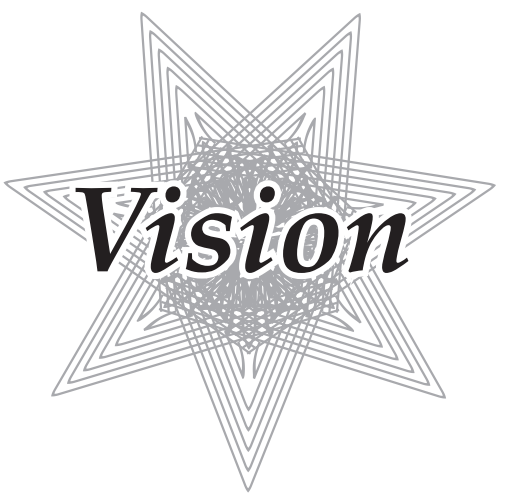

\title{
Electrochemistry and the Secret of Life
}

When we open any biochemistry textbook, we sure are greeted by a dizzily complicated network of chemical reactions, ranging from a variety of famous metabolic cycles to multistep biosynthesis pathways of key molecules. On first sight, these appear to bluntly refuse our ready comprehension.

The complexity is even multiplied by the fact that the stage on which biochemical events are performed is too minuscule to allow human imagination. For instance, inside a single cell with a volume of only 1 picoliter, hundreds or perhaps nearly a thousand of discrete reactions are probably taking place at a just-needed rate and just-needed timing to yield just-needed amount of products, with little if any spatial or temporal overlap. In addition, bioprocesses have been so refined, over the course of evolution for billions of years, as to attain a marvelous degree of energy saving.

Life is supported by such an exquisite chain and array of chemical reactions, whose secret we want to grasp by all means and then, if possible, to mimic for practical applications. To that end, electrochemistry no doubt holds enormous potentialities.

In the present atmosphere on this planet, many oxygen-respiring organisms naturally acquire energy via oxidation of high-energy organic compounds (sugars, lipids, proteins etc.) to maintain body temperature, to induce and control chemical reactions, and to do physical motions. In other words, life is in general driven by spontaneous or downhill redox reactions. Further, the medium for biochemical reactions is normally an electrolyte solution, where ions play a central role in neural signal transmission, protein association, and homeostasis, just to name a few.

Evidently these all are in the realm of electrochemistry. A half reaction of a redox process can be probed quickly and often quantitatively with an appropriate electrochemical setup by measuring electric current, as has for years been applied to highly selective sensing and chromatographic detection of bio-molecules. The ionic atmosphere can be probed by an electrochemical device that generates a signal in the form of a potential shift. Nowadays even biomolecules in a single cell are successfully detected and quantified electrochemically by use of micro-fabricated devices.

We can expect that electrochemical approaches further help unravel and elucidate biochemical mechanisms one after another in the future.

Most of the high-energy compounds noted above, in turn, originate in photosynthesis by plants and algae, where photon energy from the sun is transformed into the chemical energy of organic compounds, with simple and stable molecules of carbon dioxide and water as raw materials. A moment's reflection tells us that our food is totally the direct or indirect product of photosynthesis.

Photosynthesis enjoys an amazingly high efficiency: from photon absorption to glucose production, each of the ca. 40-step energy/electron transfers proceeds with an average quantum yield of $99 \%$ or higher. The molecular level mechanism, still remaining behind a thick wall over a number of facets, can be explored by electrochemical means, since the whole process is in principle a chain of uphill redox reactions.

With a conviction that the secret should lie in an ultrafine redox potential tuning among electron transfer components, as well as their delicate mutual arrangements, I have been engaged for more than a decade in redox potential determination of several key components, to obtain pieces of new information on the primary process of photosynthesis.

So far we electrochemists have only scratched the surface of a wealthy kingdom of biological mechanisms, and I do believe it awaits the challenge by new-especially young-people. Please forgive me for serving my own interests, but I am really happy if you join in a quest for the secret of life, including photosynthesis.

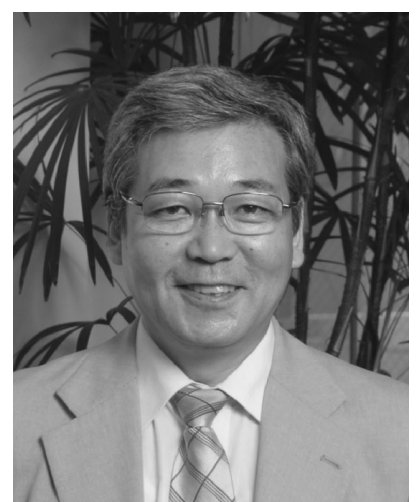

Tadashi WATANABE

Institute of Industrial Science, the University of Tokyo 